J. Lake Sci.(湖泊科学), 2018, 30(4): 937-947

DOI 10. 18307/2018. 0407

(c) 2018 by Journal of Lake Sciences

\title{
东洞庭湖沉积物覆水后磷形态变化及其释放量”
}

\author{
王 婷 ${ }^{1,2}$, 王 坤 ${ }^{1,2}$, 姜 霞 ${ }^{1,2 * *}$ \\ (1: 中国环境科学研究院, 湖泊水污染治理与生态修复技术国家工程实验室, 北京 100012) \\ (2: 中国环境科学研究院,环境基准与风险评估国家重点实验室,北京 100012)
}

\begin{abstract}
摘 要: 研究干燥覆水后低流速条件下东洞庭湖沉积物中磷的形态变化及释放量, 可以为轻度富营养化湖泊中磷的生物 地球化学循环提供基础数据, 为季节性湖泊內源营养盐的迁移转化规律研究、内源营养盐的释放风险评价提供理论依据. 本文采集处于干湿交替状态的东洞庭湖表层沉积物, 利用室内模拟装置, 研究风干沉积物低流速条件下覆水后沉积物及 上覆水中磷的形态变化. 结果表明, 低流速覆水后东洞庭湖沉积物中的磷向上覆水及大气中迁移释放, 上覆水中磷的释 放量随覆水时长增大, 释放速率随覆水时长减小, 上覆水流速和磷释放量相关性显著. 上覆水循环过程中释放到上覆水 中的溶解态有机磷比溶解态活性磷更容易吸附于颗粒物而转化为颗粒态磷. 覆水后沉积物中各形态有机磷、无机磷及磷 的生物有效性均发生转变, 覆水初期沉积物中无机磷向有机磷转化, 磷的生物可利用性增大; 上覆水循环过程中有机磷 向无机磷转化, 磷的生物可利用性减小; 覆水后沉积物的无机磷的主要组分由铝磷转变为铁磷, 有机磷的主要组分有从 中活性有机磷向活性有机磷转变的趋势.
\end{abstract}

关键词: 洞庭湖; 磷形态; 释放量; 干湿交替;沉积物

\section{Influence of rewetting process on distribution and release of phosphorus in sediments of East Lake Dongting}

\author{
WANG Ting ${ }^{1,2}$, WANG Kun ${ }^{1,2}$ \& JIANG Xia ${ }^{1,2 * *}$ \\ (1: National Engineering Laboratory of Lake Water Pollution Control and Ecological Restoration Technology, Chinese Re- \\ search Academy of Environmental Sciences, Beijing 100012, P.R.China) \\ (2: State Key Laboratory of Environmental Criteria and Risk Assessment, Chinese Research Academy of Environmental Sci- \\ ence, Beijing 100012, P.R.China)
}

\begin{abstract}
Fractions and release amount of phosphorus in sediment from East Lake Dongting were analyzed in the process of rewetting with low flow velocities, to provide evidence for phosphorus biogeochemical recycle, risk assessment of endogenous phosphorus release and eutrophication control scheme in seasonal lakes. In this paper, sediments collected in Eastern Dongting were air dried before rewetted. Phosphorus fractions in sediments and water were analyzed and indicated that phosphorus in sediments transported into water and air during rewetting process. Release amount of phosphorus in sediments increased and release rate decreased during the rewetting process. Significant positive correlation was observed between phosphorus release amount and water velocity. Dissolved organic phosphorus had relatively stronger absorption ability onto suspended particulate matter, thus they transformed into particle phosphorus more quickly than dissolved reactive phosphorus. Inorganic phosphorus in sediments transformed into organic phosphorus, and bioavailable phosphorus in sediments increased in the beginning of rewetting process. However, in the following stage of rewetting process, organic phosphorus in sediments transformed to inorganic phosphorus, and bioavailable phosphorus decreased. The dominate component of inorganic phosphorus in sediments transformed from aluminum-bound phosphate to iron-bound phosphorus. And the dominate component of organic phosphorus transformed from moderately labile organic phosphorus to labile organic phosphorus in rewetting process.
\end{abstract}

Keywords: Lake Dongting; phosphorus fractions; phosphorus release; drying-rewetting process; sediment

* 国家自然科学基金项目 (41503101,41403102) 和国家重点基础研究发展计划 (2012CB417004) 联合资助. 201710-09 收稿;2017-12-08 收修改稿. 王婷( 1985 ), 女, 博士; E-mail: wangting_btt@163.com.

** 通信作者; E-mail: jiangxia@ craes.org.cn. 
洞庭湖 $\left(28^{\circ} 44^{\prime} \sim 29^{\circ} 35^{\prime} \mathrm{N}, 111^{\circ} 53^{\prime} \sim 113^{\circ} 05^{\prime} \mathrm{E}\right.$, 图 1) 是位于长江中游的大型通江湖泊, 由西洞庭湖、南 洞庭湖和东洞庭湖组成. 由于其水文特征, 洞庭湖中长期处于枯水期与平水期出露、丰水期淹没 (干湿交替) 状态的沉积物面积占丰水期湖面积的 $60 \%$ 以上 ${ }^{[1]} .1986-2015$ 年的监测数据显示, 洞庭湖水中的主要污染 物为总氮 $(\mathrm{TN})$ 和总磷 $(\mathrm{TP})$, 其浓度已满足藻类生长需求. 洞庭湖有从中营养型湖泊向富营养性湖泊转变的 趋势, 东洞庭湖已达到轻度富营养化水平 ${ }^{[2]}$. 洞庭湖沉积物中的 TP 含量为 $357.74 \sim 998.25 \mathrm{mg} / \mathrm{kg}$, 其中东洞 庭湖沉积物的 TP 含量最高 ${ }^{[3]}$. 磷 $(\mathrm{P})$ 是湖泊富营养化发生的限制因子, 而湖泊中的 P 绝大部分存在于沉积 物中, $\mathrm{P}$ 在沉积物一水界面上的迁移转化是其在水体中循环的主要方式. 且随着 $\mathrm{P}$ 外源输人的逐步控制, 沉 积物中内源 $\mathrm{P}$ 对湖泊富营养化的作用不断凸显, 研究 P 在沉积物一水界面上的迁移转化对于湖泊富营养化 控制和治理有尤为重要. 流速变化和风浪扰动等因素引起的水动力特性变化影响磷的迁移转化规律. 不同 水动力条件下, 沉积物运动方式不同, 包括静止、起动、悬浮和沉降等. 流速在临界流速以下时, 沉积物处于 静止状态, 磷主要通过扩散、吸附和解吸作用在沉积物一水界面迁移转化; 沉积物中磷的释放以界面释放为 主, 释放量较小, 释放时间长. 湖泊流速超过一定临界流速之后, 沉积物受水流剪切力作用增大, 从静止变为 起动、悬浮和沉降; 沉积物再悬浮过程中磷的释放包含沉积物中液相污染物的释放 (孔隙水的释放)、沉积物 中固相污染物的释放和再悬浮沉积物的释放 3 个过程 ${ }^{[4.5]}$. 此外, 水文条件也是影响磷在沉积物一水界面迁 移转化规律的因素, 周期性的出露和淹没 (干湿交替) 可以使沉积物发生物理、化学和生物学变化, 进而对沉 积物一水界面的 P 迁移转化产生影响. 研究表明, 风干能增加沉积物或土壤磷的吸附 ${ }^{[6-7]}$; 也有研究表明, 风 干使沉积物对 $\mathrm{P}$ 的吸附减小 ${ }^{[8-10]}$. 沉积物或土壤组成成分、理化性质和微生物群落等的差异, 是导致其覆水 后 $\mathrm{P}$ 形态变化, 以及是否向上覆水中释放 $\mathrm{P}$ 的因素.

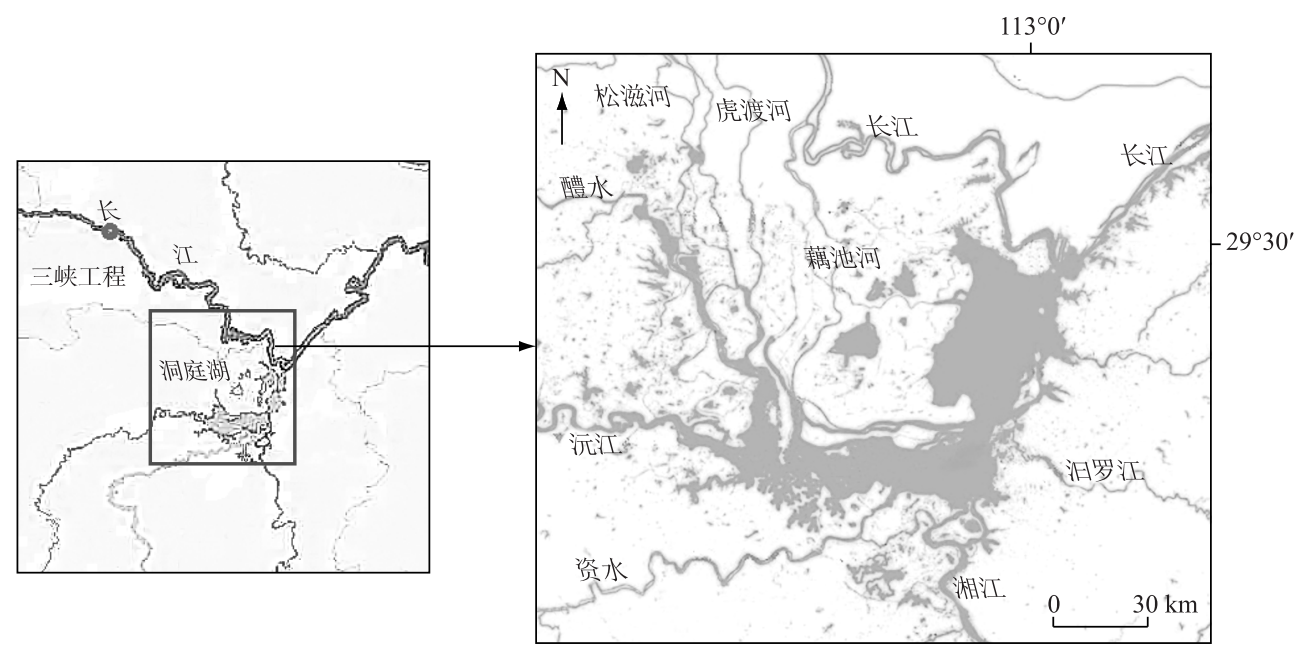

图 1 洞庭湖水系

Fig.1 Water systems in Lake Dongting

洞庭湖沉积物中 $\mathrm{P}$ 的释放通量研究表明 ${ }^{[11-12]}$, 估算方法不同, $\mathrm{P}$ 释放通量的估算结果也有很大差异, 利 用 Fick 定律计算出的 P 释放通量低于原位柱状样培养得出的 P 释放通量; 不同湖区 P 的释放通量差异也较 大 (13.505 121.18 mg/ $\left.\left(\mathrm{m}^{2} \cdot \mathrm{a}\right)\right)$, 表现为西洞庭湖>东洞庭湖>南洞庭湖. 利用 Fick 定律计算出的 P 释放通 量仅能体现沉积物孔隙度、沉积物一上覆水界面附近的营养盐浓度梯度和颗粒阻碍因子对溶解态营养盐迁 移扩散的影响. 原柱样培养实验将沉积物表面的直接释放和生物扰动的作用纳人考虑, 得出的 P 释放通量 更接近与实际释放情况; 但原位柱状样品的释放通量也不能体现紊流扩散和水平迁移扩散和干湿交替等因 素对沉积物中 P 释放通量的影响. 东洞庭湖处于轻度富营养化状态, 其沉积物中 TP 含量较高, 在洞庭湖外 源 TP 输人得到逐步控制的同时, 其內源 TP 对富营养化的影响也不容忽视. 且东洞庭湖流速较低, 大部分沉 积物处于干湿交替状态, 流速及干湿交替均会影响沉积物中 $\mathrm{P}$ 释放规律以及上覆水体的富营养化. 本文以 
东洞庭湖 P 浓度较高、流速较低、紊流扩散较低和长期处于干湿交替状态的沉积物为研究对象, 研究干燥覆 水后低流速条件下东洞庭湖 $\mathrm{P}$ 的形态变化及释放量, 可以为轻度富营养化湖泊中磷的生物地球化学循环提 供基础数据; 为洞庭湖等季节性湖泊內源营养盐的迁移转化规律研究、内源营养盐的释放风险评价、水体富 营养化及水华发生风险研究提供理论依据; 为洞庭湖等季节性湖泊的环境管理及水质改善提供理论支撑.

\section{1 研究方法}

\section{1 实验方法}

利用Van Veen 抓斗式采泥器 $\left(20 \mathrm{~cm} \times 30 \mathrm{~cm} \times 60 \mathrm{~cm}\right.$ ), 采集东洞庭湖 $\left(29^{\circ} 23^{\prime} 53.2^{\prime \prime} \mathrm{N}, 113^{\circ} 06^{\prime} 28.4^{\prime \prime} \mathrm{E}\right)$ 的 表层沉积物 $\left(<10 \mathrm{~cm}\right.$ ), 采样点水深 $1 \sim 2 \mathrm{~m}$. 将采集到的沉积物去除石块等固体杂物后混匀, $24^{\circ} \mathrm{C}$ 下风干后 备用.

将 $1 \mathrm{~kg}$ 风干的表层沉积物样品人工破碎至可过 5 目笁, 平铺人水循环模拟装置 $(100 \mathrm{~cm} \times 10 \mathrm{~cm} \times 70 \mathrm{~cm}$, 图 2) 底部, 沉积物在模拟装置中高度为 $3 \mathrm{~cm}$. 为避免沉积物再县浮, 用蠕动葲缓慢加人 $50 \mathrm{~L}$ 纯水, 上覆水高 度为 $55 \mathrm{~cm}$. 上覆水分别以不同流速 $(0.025 、 0.05 、 0.1 、 0.15$ 和 $0.2 \mathrm{~cm} / \mathrm{s})$ 在人工气候室中循环 $60 \mathrm{~d}$. 覆水循环 过程中, 光照时长为 $12 \mathrm{~h} / \mathrm{d}$, 温度为 $25^{\circ} \mathrm{C}$. 蒸发水量由纯水进行补充. 覆水时长分别为 $0 、 1 、 12 、 30$ 和 $60 \mathrm{~d}$ 时 采集 $2 \mathrm{~g}$ 沉积物样品和 $100 \mathrm{ml}$ 中层水样进行各形态磷的检测.

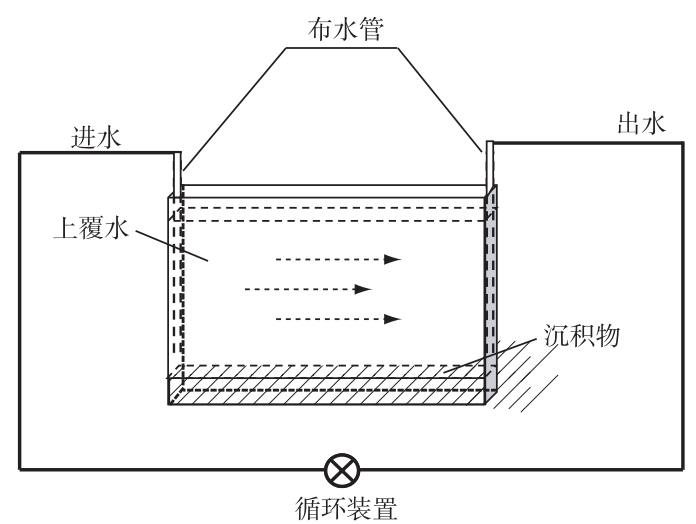

图 2 水循环模拟装置

Fig. 2 Hydrological unit with water flow

\section{2 测试方法}

水样中 $\mathrm{pH}$ 、溶解氧 (DO) 和氧化还原电位 ( ORP) 分别用 HACH pH/溶解氧/电导率仪及 Mettler Toledo SG68-SevenGo 多通道水质检测仪进行检测. 沉积物中 $\mathrm{pH}$ 和 ORP 用笔式余氯计进行检测. 水样中的 TP、溶 解态总磷 (DTP) 及溶解态活性磷 (DRP) 浓度分别采用磷钼蓝分光光度法、过 $0.45 \mu \mathrm{m}$ 滤膜一磷钼蓝分光光 度法测定和 $\mathrm{K}_{2} \mathrm{~S}_{2} \mathrm{O}_{8}$ 高温高压消解一磷钼蓝分光光度法测定 ${ }^{[13]}$.

沉积物中的总磷 (TP) 含量采用 SMT 法进行测定 ${ }^{[14]}$. 对沉积物中不同形态的 P( 可交换磷、自生钙磷、铁 磷、铝磷、闭蓄磷和残渣磷) 进行连续提取 ${ }^{[15]}$, 提取液中的无机磷 ( IP) 和 TP 含量分别采用磷钼蓝分光光度 法和 $\mathrm{K}_{2} \mathrm{~S}_{2} \mathrm{O}_{8}$ 高温高压消解一磷钼蓝分光光度法测定. 根据提取液中测出的 IP 含量, 计算得出沉积物中可交 换磷、自生钙磷、铁磷、铝磷和闭蓄磷含量. 根据提取液中测出的 IP 和 TP 含量, 得出提取液中有机磷 ( OP = TP-IP) 含量, 并计算出沉积物中的可交换有机磷、自生钙有机磷、铁有机磷、铝有机磷、闭蓄有机磷和残渣有 机磷, 沉积物中活性有机磷、中活性有机磷、中稳态及高稳态有机磷含量计算方法如下 ${ }^{[16]}$ :

活性有机磷 $=$ 可交换有机磷 + 铁有机磷

中活性有机磷 $=$ 自生钙有机磷 + 闭蓄有机磷

中稳态及高稳态有机磷 $=$ 铝有机磷 + 残渣有机磷

沉积物中溶解态二价铁 $\left(\mathrm{Fe}^{2+}\right)$ 和总铁含量采用邻菲罗啉分光光度法进行测定, 计算得出三价铁 $\left(\mathrm{Fe}^{3+}\right)$ 
含量 ${ }^{[16]}$.

所有样品分析均做 3 次平行, 实验数据均以 3 次样品分析的平均值表示, 误差 $\leqslant 7.32 \%$.

\section{3 释放量及释放速率计算方法}

以上覆水中的 $\mathrm{TP}$ 浓度计算得出沉积物中 $\mathrm{P}$ 的释放量 $(\Delta P)$ 及释放速率 $\left(\nu_{\mathrm{P}}\right)$ :

$$
\begin{gathered}
\Delta P=\left(T P_{t}-T P_{0}\right) \times V / Q \\
\nu_{\mathrm{P}}=\left(\Delta P_{t_{1}}-\Delta P_{t_{2}}\right) /\left(t_{1}-t_{2}\right)
\end{gathered}
$$

式中, $T P_{t}$ 为覆水时长为 $t$ 时, 上覆水中的 $\mathrm{TP}$ 浓度 $(\mathrm{mg} / \mathrm{L}) ; T P_{0}$ 为超纯水中的 $\mathrm{TP}$ 浓度 $(\mathrm{mg} / \mathrm{L}) ; V$ 为上覆水体 积 $(\mathrm{L}) ; Q$ 为沉积物质量 $(\mathrm{kg}) ; \Delta P_{t_{1}} 、 \Delta P_{t_{2}}$ 分别为覆水时长 $t_{1} 、 t_{2}$ 时的 $\Delta P(\mathrm{mg} / \mathrm{kg}) ; t_{1} 、 t_{2}$ 为覆水时长 $(\mathrm{d})$.

\section{2 结果与讨论}

\section{1 沉积物和水的理化性质}

水循环过程中, 上覆水温度、 $\mathrm{DO} 、 \mathrm{ORP}$ 和 $\mathrm{pH}$ 分别为 $25 \pm 2^{\circ} \mathrm{C} 、 6.99 \sim 8.45 \mathrm{mg} / \mathrm{L} 、 118 \sim 249 \mathrm{mV}$ 和 $7.26 \sim$ 8.44. 沉积物的 $\mathrm{pH}$ 和 ORP 分别为 7.59 8.15 和-180 180 mV. 上覆水循环过程中沉积物的 $\mathrm{pH}$ 下降, 沉积 物的 ORP 先降低后升高. 而上覆水的 $\mathrm{pH}$ 和 DO 浓度最终均有所升高 (图 3). 大部分上覆水中 ORP 先降低 后升高至 $200 \mathrm{mV}$ 以上. 循环过程中, 水中 $\mathrm{DO}$ 浓度先由 $8.07 \mathrm{mg} / \mathrm{L}$ 缓慢降低至 $7.72 \mathrm{mg} / \mathrm{L}$, 而后升高.
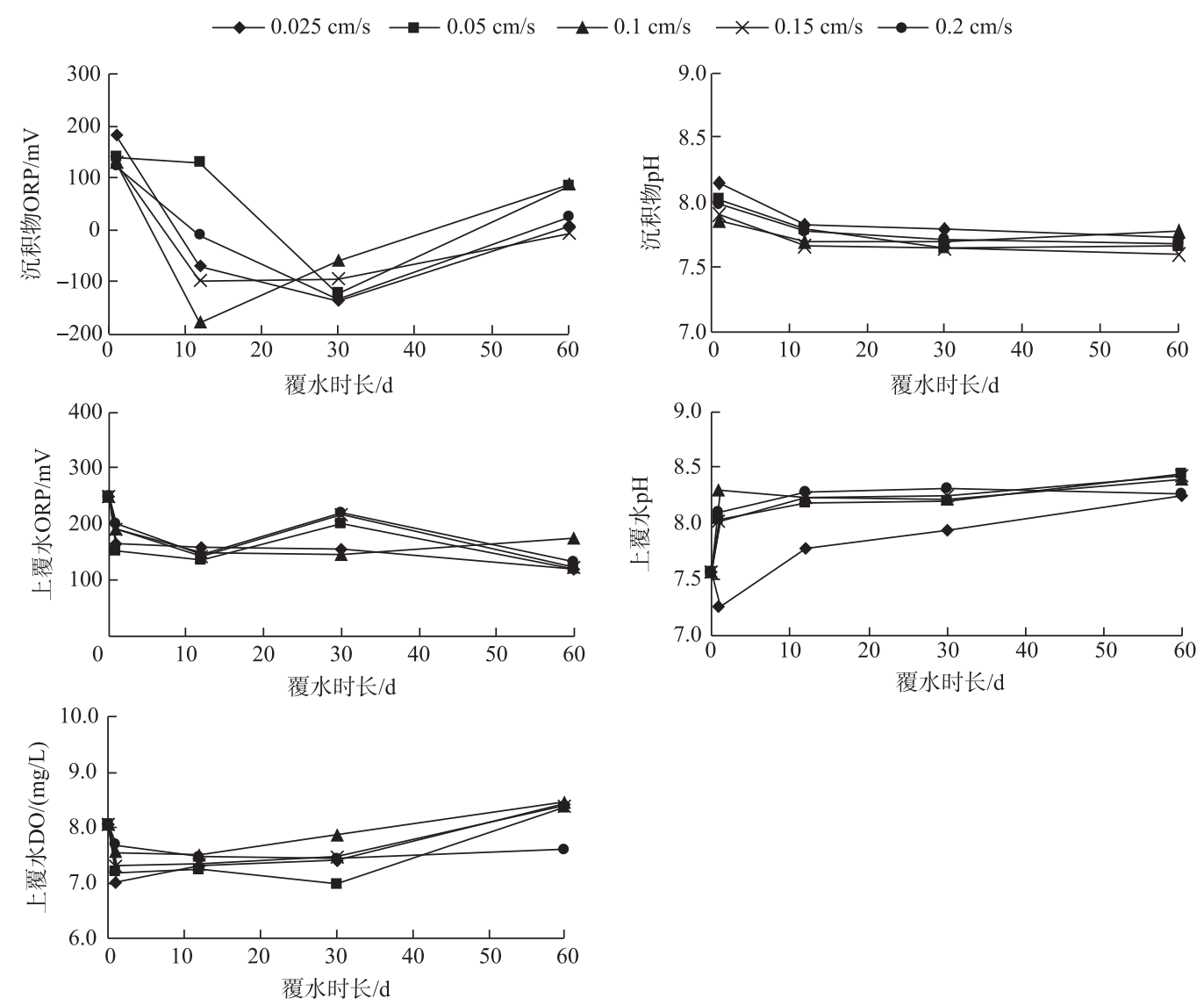

图 3 覆水过程中沉积物 $\mathrm{ORP} 、 \mathrm{pH}$ 以及上覆水 $\mathrm{ORP} 、 \mathrm{pH}$ 和 $\mathrm{DO}$ 浓度变化

Fig. 3 ORP, pH and DO in sediment and water column during rewetting process 


\section{2 上覆水中磷分布}

沉积物覆水过程中, 上覆水中 TP 浓度均呈现随覆 水时长增大的趋势, 且增大速率随时间减小; 覆水时长 为 $60 \mathrm{~d}$ 时,上覆水中 $\mathrm{TP}$ 浓度增到 $0.454 \sim 0.825 \mathrm{mg} / \mathrm{L}$, 流速较大的上覆水 $(\geqslant 0.15 \mathrm{~cm} / \mathrm{s})$ 中 $\mathrm{TP}$ 浓度较高. 上 覆水中的 DTP 浓度在覆水 $30 \mathrm{~d}$ 内先增加至 $0.264 \sim$ $0.371 \mathrm{mg} / \mathrm{L}$, 而后降低 $13.6 \% \sim 45.3 \%$. DRP 浓度持续 增大, 覆水时长为 $1 \mathrm{~d}$ 时的增大速率较大; 覆水时长为 $1 \mathrm{~d}$ 时, 流速较大的上覆水 $(0.2 \mathrm{~cm} / \mathrm{s})$ 中 DRP 浓度最 高, 是其他流速上覆水中 DRP 浓度的 2 倍以上(图 4). 上覆水流速与上覆水中 TP、DTP 及 DRP 浓度之间的 相关性不显著 $(R \leqslant 0.544)$.

\section{3 沉积物中磷形态分布}

沉积物中 TP 含量为 $625.68 \sim 876.31 \mu \mathrm{g} / \mathrm{g}$, 萃取率 为 $78.51 \% \sim 109.96 \%$. 沉积物覆水后以及覆水过程中, IP、有机磷 ( OP) 总量及形态分布均有明显变化.

覆水 $1 \mathrm{~d}$ 后, 沉积物中 IP 减少了 $81.09 \% \sim 85.55 \%$; 除可交换磷外,其他各个形态的 IP 均有不同程度的减 少 (19.98\% 99.20\%), 其中以铝磷的减少幅度最大 (98.00\% 99.20\%); 覆水前沉积物中铝磷占 IP 比例 最大 $(62.41 \%)$, 覆水后以 TIP 组分以自生钻磷为主 $(47.23 \% \sim 55.25 \%)$; 覆水 $1 \mathrm{~d}$ 后, 流速为 $0.025 \mathrm{~cm} / \mathrm{s}$ 的 沉积物中可交换磷减少了 $54.28 \%$, 而流速较大的沉积 物中 $(\geqslant 0.05 \mathrm{~cm} / \mathrm{s})$ 可交换磷在沉积物覆水后以及覆 水循环过程中均有所增多 $(20.32 \% \sim 131.38 \%)$. 上覆 水循环过程中, 沉积物的 IP 随覆水时间不断增多, 覆 水时长为 $60 \mathrm{~d}$ 时的 IP 含量是覆水时长为 $1 \mathrm{~d}$ 时 IP 含
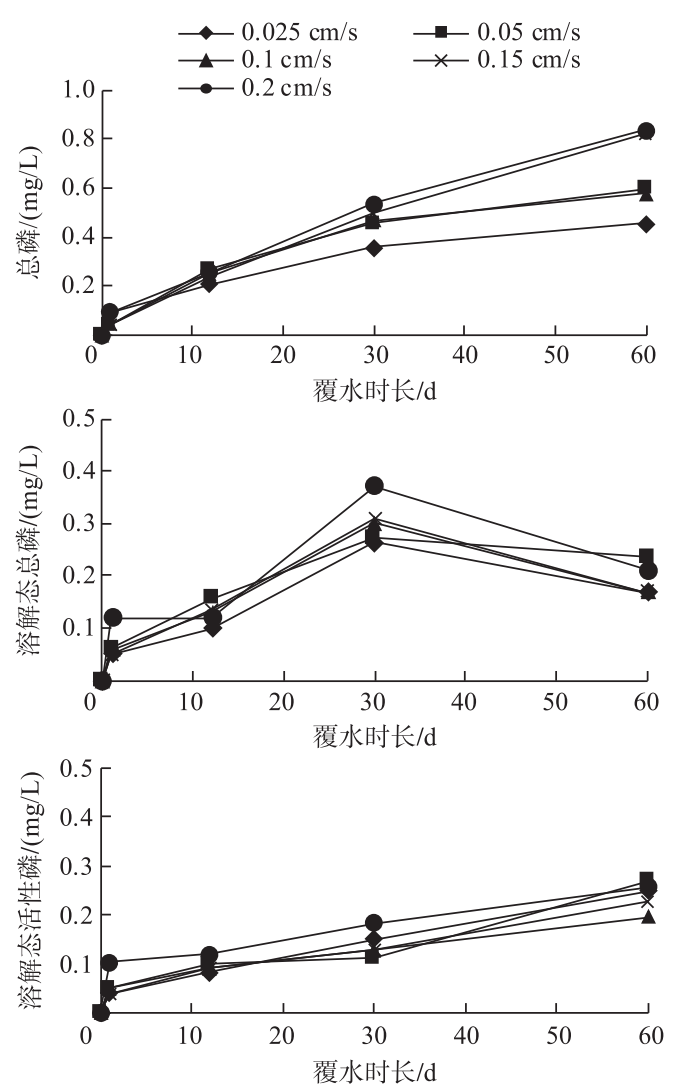

图 4 覆水过程中上覆水中磷形态变化

Fig.4 Fractions of $\mathrm{P}$ in water column during rewetting process 量的 2.73 3.37 倍; IP 的主要组分由自生钙磷逐渐转变为铁磷 ( $41.75 \% \sim 64.39 \%)$; 上覆水循环过程中, 可交 换磷、自生钲磷和铁磷增多, 覆水 $60 \mathrm{~d}$ 后增大倍数分别为 $1.12 \sim 4.45 、 1.36 \sim 2.59$ 和 $5.61 \sim 11.06$ 倍; 覆水过程 中沉积物中的铝磷和闭蓄磷整体呈现先减少后增多的趋势, 覆水时长为 $12 \mathrm{~d}$ 时, 铝磷和闭蓄磷含量最低 (图 $5)$.

覆水 $1 \mathrm{~d}$ 后, 沉积物中的有 $\mathrm{OP}$ 含量增大到覆水前的 $1.80 \sim 2.26$ 倍; 其中活性有机磷和中活性有机磷含 量均有明显增大, 分别增大了 $4.11 \sim 6.84$ 和 $1.53 \sim 2.31$ 倍; 中稳态和高稳态有机磷含量减小了 $81.45 \%$ $85.56 \%$. 上覆水循环过程中, 沉积物的有机磷有减少趋势; 其中活性有机磷含量持续增加, 不同流速的上覆 水循环过程中活性有机磷占有机磷比例从 $4.49 \%$ 7.59\% 增大到 $21.38 \% \sim 34.73 \%$; 占比例最大的中活性有 机磷含量随覆水时长减小, 占 OP 比例从 $89.19 \% \sim 93.30 \%$ 降低至 $53.51 \% \sim 67.06 \%$; 中稳态和高稳态有机磷 含量整体有增大的趋势.

由于沉积物中的可交换磷、铁磷、铝磷、活性有机磷和中活性有机磷在不同条件下均可能以溶解态磷酸 盐的形态释放, 而被藻类生长所吸收利用 ${ }^{[17-22]}$, 沉积物中的生物可利用磷 $(\mathrm{BAP})=$ 可交换磷 + 铁磷 + 铝磷 + 活 性有机磷+中活性有机磷 ${ }^{[15]}$. 干燥沉积物覆水 $1 \mathrm{~d}$ 后, BAP 含量增大 $30 \%$ 以上, 而后随覆水时长减小 (图 5).

\section{4 磷的迁移转化}

根据沉积物及上覆水中的 TP, 计算得出沉积物一水体系中 P 总量 (S-WP) 为 $1843 \sim 2552 \mathrm{mg}$. 在覆水时 长不变的条件下, S-WP 与流速之间不存在显著相关性 $(R=-0.025)$. 不同覆水时长下, 平均 S-WP 呈现先升 高后降低的趋势 (图 6). 沉积物覆水后平均 S-WP 有少量增大 (3.50\%), 而随着覆水时长的增加, 平均 S-WP 

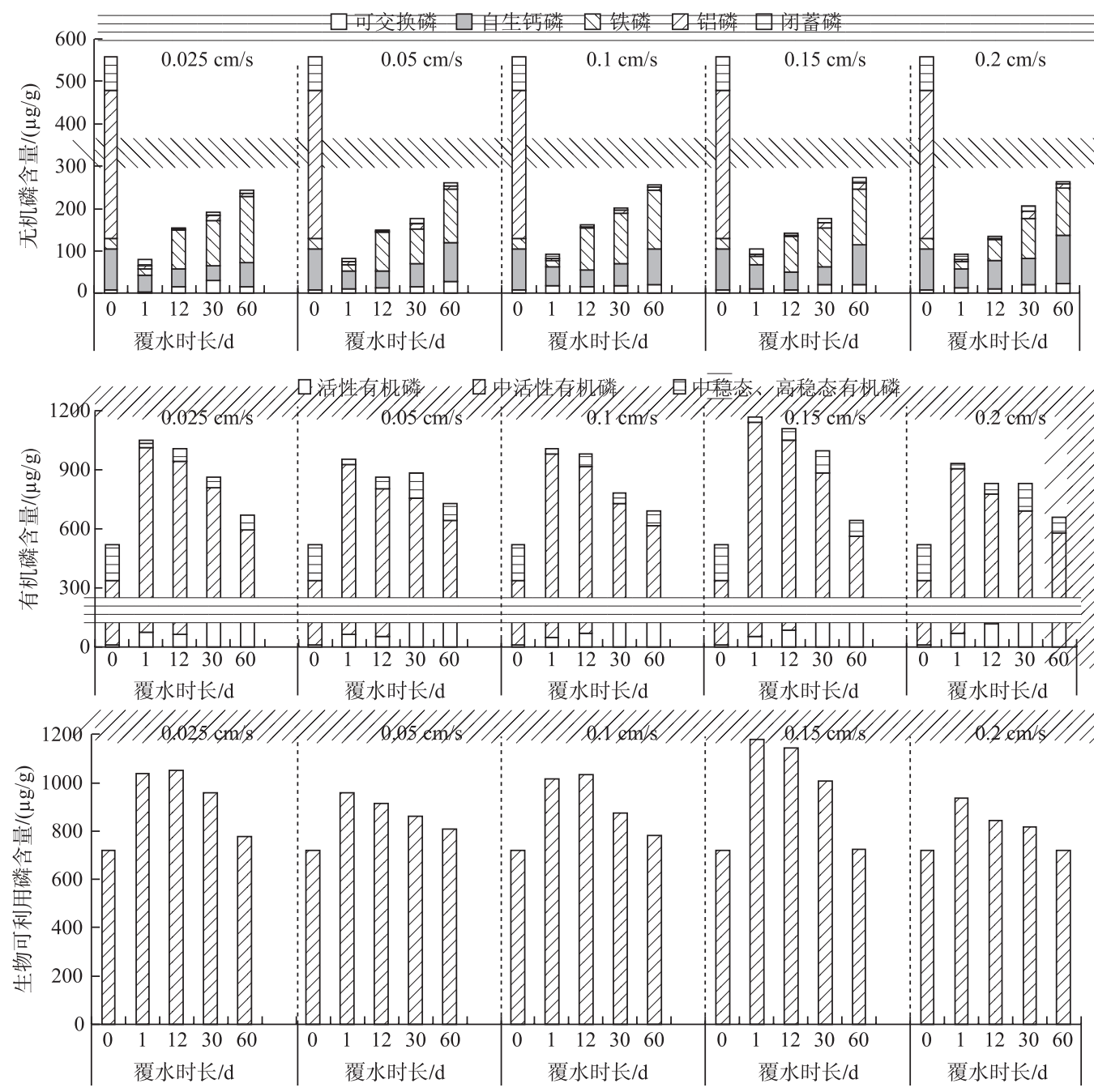

图 5 覆水过程中沉积物无机磷、有机磷和生物可利用磷形态分布

Fig.5 Fractions of inorganic $\mathrm{P}$, organic $\mathrm{P}$ and bioavailable $\mathrm{P}$ in sediments during rewetting process

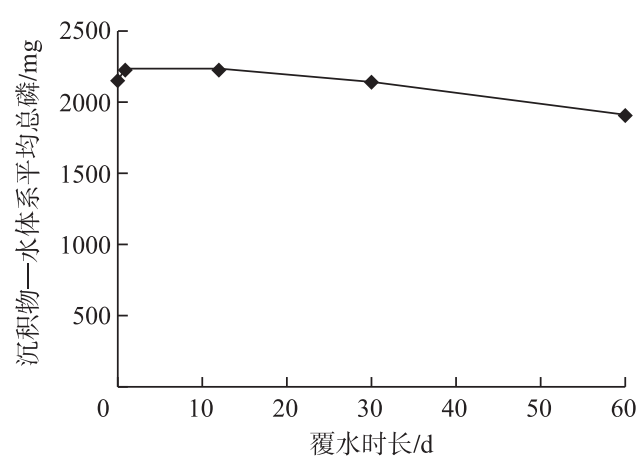

图 6 覆水过程中沉积物一水体系中平均总磷

Fig.6 Average total amount of $\mathrm{P}$ in sedimentwater system during rewetting process
有所减小; 覆水 $60 \mathrm{~d}$ 时平均 S-WP 为覆水前的 $88.51 \%$. 覆水后沉积物中的 $\mathrm{P}$ 向上覆水迁移释放, $\mathrm{P}$ 的释放量随 覆水时长不断增大, 而释放速率随覆水时长不断减小. 覆水时长为 $60 \mathrm{~d}$ 时, 上覆水中 $\mathrm{TP}$ 占 $\mathrm{S}-\mathrm{WP}$ 的 $1.23 \% \sim$ $2.21 \%$. 覆水 $60 \mathrm{~d}$ 时, 磷的释放量 $\Delta \mathrm{P}$ 为 $11.36 \sim 20.93$ $\mathrm{mg} / \mathrm{kg}$, 释放速率 $\nu_{\mathrm{P}}$ 为 $1.58 \sim 5.44 \mathrm{mg} /\left(\mathrm{m}^{2} \cdot \mathrm{d}\right)$. 孙士权 等 ${ }^{[23]}$ 的研究表明, 静置覆水时长为 $7 \mathrm{~d}$ 时, 东洞庭湖沉 积物的磷释放量为 $3.72 \mathrm{mg} / \mathrm{kg}$, 最大释放速率为 48.6 $\mathrm{mg} /\left(\mathrm{m}^{2} \cdot \mathrm{d}\right)$, 与本研究覆水时长为 $12 \mathrm{~d}$ 时的磷释放量 $(5.16 \sim 6.71 \mathrm{mg} / \mathrm{kg})$ 及最大释放速率 $(21.67 \sim 47.47 \mathrm{mg} /$ $\left.\left(\mathrm{m}^{2} \cdot \mathrm{d}\right)\right)$ 相近 (图 7). 而本研究得出的覆水 $60 \mathrm{~d}$ 时 $\mathrm{P}$ 的 释放速率比 Liang 等 ${ }^{[12]}$ 采用 DGT 技术得出的持续覆水 
的洞庭湖沉积物 $\mathrm{P}$ 释放速率 $\left(0.037 \sim 0.332 \mathrm{mg} /\left(\mathrm{m}^{2} \cdot \mathrm{d}\right)\right)$ 大 1 个数量级. 由此可见,干湿交替过程可促进东洞 庭湖中 $\mathrm{P}$ 的释放. 在 $99 \%$ 的置信度水平下, 上覆水流速与 $\Delta \mathrm{P}$ 呈显著相关 $(R=0.9306)$. 覆水后沉积物中 IP 向 OP 转化, 各形态 P 向中活性有机磷转化; 上覆水循环过程中, 沉积物中 OP 向 IP 转化, 中活性有机磷向其 他形态 $\mathrm{P}$ 转化.

从沉积物一水体系中 $\mathrm{P}$ 质量守恒的角度可以得出, 覆水后沉积物中的磷化氢 (MBP) 转化为 IP, 可能是 导致覆水初期部分 S-WP 增大的原因. 而随着覆水时长的不断增大, 沉积物中的 $\mathrm{P}$ 又通过厌氧微生物转化为 MBP 释放到大气中, 使得 S-WP 减小. 湖泊是 MBP 的 “源” 和 “汇”, 湖泊中的 MBP 主要吸附于沉积物及悬浮 颗粒物 ${ }^{[24-26]}$, 湖泊也是大气中 MBP 的主要来源之一 ${ }^{[26]}$. MBP 的产生机理还没有明确研究结论, 但有研究表 明, MBP 可能来源于戻氧微生物对 $\mathrm{OP}$ 的降解 ${ }^{[27-28]}$, 而在本研究中 S-WP 的增大和减小, 即 MBP 的转化及释 放, 也与沉积物中 $\mathrm{OP}$ 含量存在显著相关性 $(R=0.8067)$. 沉积物中 MBP 可以通过扰动等因素进人上覆 水 $^{[29]}$,而由于其强还原性,进人上覆水中的部分 $M B P$ 也可以转化为 DRP 及其他形态 $\mathrm{P}^{[30]}$.

覆水过程中, 沉积物不断向上覆水释放 DTP ( DOP、DIP 及 MBP), 使上覆水中的 TP、颗粒态总磷 (PP) 和 DRP 持续增多, DTP 先增多后减少. 有机磷的吸附解吸研究表明 ${ }^{[31]}, \mathrm{DOP}$ (植酸、甘油磷酸等) 在铁铝氧化物 上的吸附量低于 DRP, 但 DOP 在铁铝氧化物上的解吸率和解吸速率低于 DRP. 因此, DOP 和 DRP 在颗粒物 上的吸附解吸规律的差异, 可能是上覆水循环过程中, DOP 及 DRP 浓度变化趋势不同, DOP 更容易吸附于 颗粒物并转化为 PP 的原因. 研究表明 ${ }^{[32-33]}$, PP 是上覆水中 TP 的主要组成, 其浓度受径流输沙量或悬浮颗 粒物含量影响较大. PP 的主要存在形式是颗粒态无机磷 (PIP), PIP 的解吸释放是水体中 DRP 的动态补充; 颗粒态有机磷 (POP) 的组分及其在 PP 中所占的比例与湖泊类型有关, 人为干扰较少和生态系统稳定的湖 泊中 POP 在 PP 中所占比例较高, POP 中的磷酸单酯可降解为 DRP 并被浮游植物利用 ${ }^{[33-34]}$.
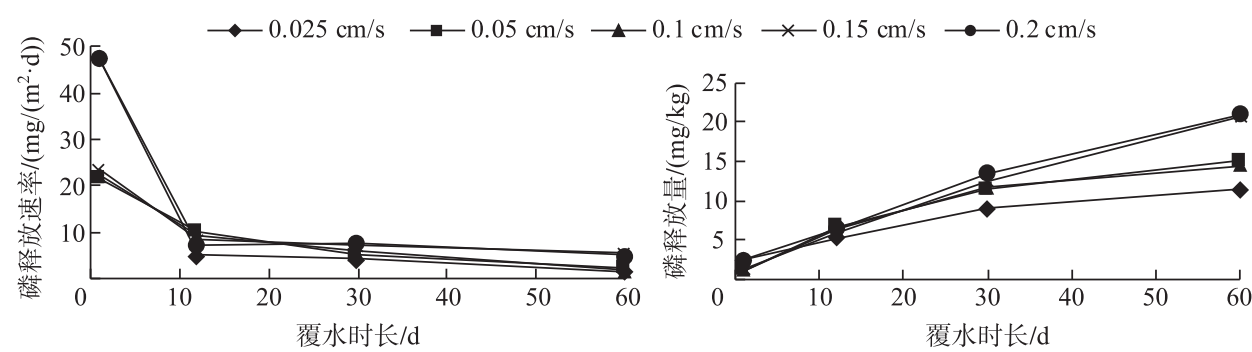

图 7 覆水过程中沉积物磷释放量

Fig.7 Phosphorus release amount during rewetting process

无机磷中的铁磷、铝磷是沉积物 “源” “汇” 转化过程中最活跃的磷组分 ${ }^{[35]}$, 干燥沉积物覆水过程中铁 磷、铝磷的转化行为和其他无机磷组分相比也更为活跃. (1)覆水后沉积物的 ORP 降低, $\mathrm{Fe}^{3+}$ 还原为 $\mathrm{Fe}^{2+}$ (图 8), 氢氧化铝溶解度增加, 铁磷及铝磷释放; (2)覆水后沉积物的 $\mathrm{pH}$ 值增大, $\mathrm{OH}^{-}$与 $\mathrm{PO}_{4}^{3-}$ 竞争吸附点位, 导致 铁磷及铝磷释放; (3)沉积物干燥过程中无定型铁氧化物形成结晶度更高的铁氧化物, 对磷的吸附能力减 弱 ${ }^{[36-39]}$, 也促进铁磷释放. 研究表明, 即使沉积物的 ORP 降至 $-400 \mathrm{mV}$, 沉积物中仍然存在大量 $\mathrm{Fe}^{3+}$, 并对磷 进行有效吸附 ${ }^{[40]}$; 而上覆水循环过程中, 沉积物 $\mathrm{ORP}$ 有所回升, $\mathrm{pH}$ 下降, 上覆水处于好氧条件下, 铁磷含量 有所增大. 沉积物中的 $\mathrm{Fe}$ 和 Mn 均是氧化还原敏感性元素, 且铁、锰氧化物可充当有机质降解的电子接收 体; 此外, 镇氧化物还可与 $\mathrm{Fe}^{2+}$ 反应 ${ }^{[41]}: 2 \mathrm{Fe}^{2+}+\mathrm{MnO}_{2}+4 \mathrm{H}_{2} \mathrm{O} \rightarrow \mathrm{Mn}^{2+}+2 \mathrm{Fe}(\mathrm{OH})_{3}+2 \mathrm{H}^{+}$, 使沉积物中的 $\mathrm{Fe}$ 主要 以 $\mathrm{Fe}^{3+}$ 形式存在, 吸附水体中磷, 抑制磷释放. 沉积物中自生钙磷主要为由于生物作用沉积的磷 (碳酸钻、羟 基磷灰石等), 沉积物覆水后的偏碱性环境 ( $\mathrm{pH}=7.91 \sim 8.15$, 图 3) 是自生䥾磷释放的原因 ${ }^{[42]}$; 上覆水循环过 程中, 微生物的增加促使沉积物中自生钲磷的蓄积增多 ${ }^{[43]}$. 沉积物中的闭蓄磷主要为难溶的氧化铁铝胶膜 包裹的磷, 干燥沉积物覆水后沉积物的氧化还原条件发生变化, 破坏氧化铁铝胶膜, 是闭蓄磷的释放的原因.

干湿交替条件变化促进沉积物中微生物多样性及群落结构变化, 可能是造成干燥沉积物覆水后 $\mathrm{OP}$ 含 量增大的原因. 有机磷中的中活性有机磷 (植酸钻) 占有机磷的 $54.70 \% \sim 94.17 \%$, 以磷酸酯、磷脂、核酸、磷 


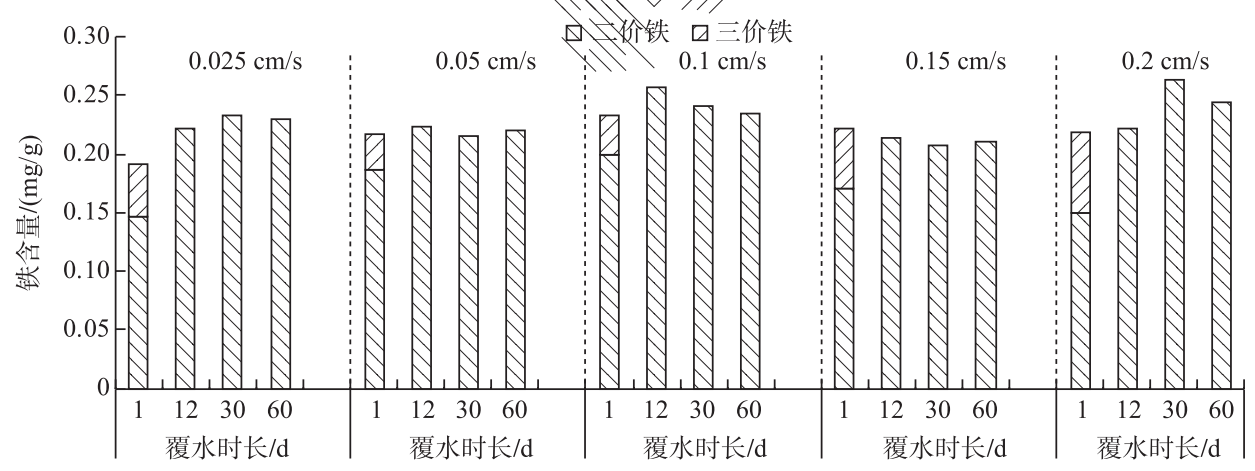

图 8 覆水过程中沉积物溶解性铁含量

Fig.8 Dissolved iron content in sediments during rewetting process

蛋白和磷酸糖类为主 ${ }^{[44]}$, 均为易分解的生物大分子, 稳定性差, 在一定条件下可分解或矿化为活性有机磷或 其他形态 $\mathrm{P}^{[45]}$.

沉积物中 P 的生物有效性和多个形态的 P 有关 $(\mathrm{BAP}=$ 可交换磷+铁磷+铝磷+活性有机磷+中活性有机 磷), 单个形态 $\mathrm{P}$ 的增大或减小并不足以体现 $\mathrm{P}$ 的生物有效性. 干燥沉积物覆水后, 虽然可交换磷、铁磷和铝 磷含量减小, 但随着活性有机磷和中活性有机磷含量的增大, 沉积物中 P 的生物有效性在短期内有所增大. 而后随着覆水时长的增加, 沉积物中活性有机磷和中活性有机磷含量的减小, $\mathrm{P}$ 的生物有效性也随之减小. 因此,微生物群落结构变化对沉积物中 P 生物有效性的影响较大.

\section{3 结论}

1) 覆水使沉积物中磷形态发生转化, 覆水初期沉积物中磷化氢向无机磷转化, 无机磷向有机磷转化; 上 覆水循环过程中有机磷向无机磷及磷化氢转化, 磷化氢释放到大气中造成沉积物一水体系中磷总量有所 降低.

2) 覆水后沉积物的 $\mathrm{pH}$ 和氧化还原条件变化是无机磷各组分释放的主要因素, 无机磷的主要组分由自 生钻磷逐渐转变为铁磷. 覆水后微生物群落变化是有机磷组分变化的主要因素, 有机磷的主要组分有从中 活性有机磷向活性有机磷转变的趋势.

3) 覆水后磷的释放量随覆水时长不断增大, 释放速率随覆水时长不断减小, 上覆水流速和磷释放量相 关性显著. 上覆水循环过程中处于有氧条件下, 释放到上覆水中的溶解态有机磷比溶解态活性磷更容易吸 附于颗粒物而转化为颗粒态磷.

4) 覆水后沉积物中的生物可利用磷在覆水初期有所增大, 随覆水时间减小. 微生物群落结构变化对沉 积物中磷的生物有效性影响较大.

5) 干湿交替促进沉积物中磷向上覆水中以及大气中释放. 沉积物作为内源磷向上覆水中的释放增加上 覆水的富营养化以及水华发生风险. 不同水动力条件对沉积物內源磷向大气中释放的影响, 洞庭湖中磷化 氢的释放通量还有待深人研究.

\section{4 参考文献}

[ 1 ] Ke WL, Chen CZ, Ji HX et al. A loop-like relationship between water surface area of Lake Dongting and water level at Chenglingji, the Yangtze River. J Lake Sci, 2017, 2017, 29(3) : 753-764. DOI: 10.18307/2017.0325. [柯文丽, 陈成 忠, 吉红霞等. 洞庭湖水面面积与城陵矶水位之间的绳套关系. 湖泊科学, 2017, 29(3) : 753-764.]

[ 2 ] Xiong J, Yu FQ, Tian Q et al. The evolution of water quality and nutrient condition in Lake Dongting in recent 30 years. $J$ Lake Sci, 2016, 28(6) : 1217-1225. DOI: 10.18307/2016.0607. [ 熊剑, 喻方琴, 田琪等. 近 30 年来洞庭湖水质营养 状况演变特征分析. 湖泊科学, 2016, 28(6): 1217-1225.]

[ 3 ] Wang Y, Jiang X, Li YF et al. Spatial and temporal distribution of nitrogen and phosphorus and nutritional characteristics 
of water in Dongting Lake. Research of Environmental Sciences, 2014, 27(5): 484-491. [王岩, 姜霞, 李永峰等. 洞庭 湖氮磷时空分布与水体营养状态特征. 环境科学研究, 2014, 27(5) : 484-491.]

[ 4 ] Xiao Y, Cheng HK, Tang HW et al. Review of influence of hydrodynamic action on distribution of pollutants in water and sediment in river. Journal of Hohai University: Natural Sciences, 2015, 43(5) : 480-487. [ 肖洋, 成浩科, 唐洪武等. 水 动力作用对污染物在河流水沙两相中分配的影响研究进展. 河海大学学报: 自然科学版, 2015, 43 (5): 480-487.]

[ 5 ] Wan J, Wang Z, Li Z et al. Critical velocity in phosphorus exchange processes across the sediment-water interface. Journal of Environmental Sciences, 2013, 25(10): 1966-1971.

[ 6 ] Peltovuori T, Soinne H. Phosphorus solubility and sorption in frozen, air-dried and field-moist soil. European Journal of Soil Science, 2005, 56(6) : 821-826.

[ 7 ] Hoffmann CC, Heiberg L, Audet J et al. Low phosphorus release but high nitrogen removal in two restored riparian wetlands inundated with agricultural drainage water. Ecological Engineering, 2012, 46: 75-87.

[ 8 ] Watts C. Seasonal phosphorus release from exposed, re-inundated littoral sediments of two australian reservoirs. Hydrobiologia, 2000, 431(1): 27-39.

[ 9 ] Xiao WJ, Song CL, Cao XY et al. Effects of airdrying on phosphorus sorption in shallow lake sediment, China. Fresenius Environmental Bulletin, 2012, 21: 672-678.

[10] Schönbrunner IM, Preiner S, Hein T. Impact of drying and re-flooding of sediment on phosphorus dynamics of river-floodplain systems. Science of the Total Environment, 2012, 432 : 329-337.

[11] Gao Y, Liang T, Tian S et al. High-resolution imaging of labile phosphorus and its relationship with iron redox state in lake sediments. Environmental Pollution, 2016, 219: 466-474.

[12] Liang T, Tong Y, Wang X et al. Release of reactive phosphorus from sediments in Dongting Lake linked with the Yangtze River. Environmental Chemistry, 2016, 14(1) : 48-54.

[13] Jiang X, Wang SH eds. Handbook on sediment quality survey and assessment. Beijing: Science Press, 2012. [姜霞, 王 书航. 沉积物质量调查评估手册. 北京: 科学出版社, 2012.]

[14] Ruban V, López-Sánchez JF, Pardo P et al. Harmonized protocol and certified reference material for the determination of extractable contents of phosphorus in freshwater sediments-a synthesis of recent works. Fresenius J Anal Chem, 2001, 370: 224-228.

[15] Yang B, Wang T, Wang K et al. A modified sequential extraction method for the determination of phosphorus fraction in sediment. Environmental Science \& Technology, 2017, 40(9): 90-97. [杨斌, 王婷, 王坤等. 一种改进的磷形态连续 提取方法. 环境科学与技术, 2017, 40(9): 90-97.]

[16] Ministry of Environmental Protection of the People's Republic of China, Editorial Board of Water and Wastewater Monitoring and Analysis Methods ed. Water and wastewater monitoring and analysis methods: 4th edition. Beijing: China Environmental Science Press, 2002. [国家环境保护总局《水和废水监测分析方法》编委会. 水和废水监测分析方法: 第 4 版. 北京: 中国环境科学出版社, 2002.]

[17] Yu KC, Tsai LJ, Chen SH et al. Chemical binding of heavy metals in anoxic river sediments. Water Res, 2001, 35(17) : 4086-4094.

[18] Weng HX. Binding state of phosphorus in river sediment and its environmental geochemical significance. Chinese Science Bulletin, 1993, 38(13) : 1219-1222. [翁焕新. 河流沉积物中磷的结合状态及其环境地球化学意义. 科学通报, $1993, \mathbf{3 8}(13): 1219-1222$.

[19] Andrieux F, Aminot A. A two-year survey of phosphorus speciation in the sediments of the Bay of Seine (France). Continental Shelf Research, 1997, 17(10): 1229-1245.

[20] Hua ZZ, Zhu XQ, Wang XR. Study on bioavailability of Selenastrum capricornutum influenced by released phosphorus. Acta Science Circumstantiae, 2000, 20(1): 100-105. [华兆哲, 朱晓青, 王晓蓉. 太湖沉积物磷释放对羊角月芽藻的生 物可利用性研究. 环境科学学报, 2000, 20(1): 100-105.]

[21] Wu P. Recent sedimentary records of primary production and nutrients in the typical areas of the East China Sea and the Yellow Sea [Dissertation]. Qingdao: Ocean University of China, 2007. [吴鹏. 东、黄海典型海域初级生产力和氮、磷 营养要素的近代沉积记录 [学位论文]. 青岛: 中国海洋大学, 2007.]

[22] Xiong SG, Xi BD, Wei D et al. Distribution of different phosphorus fractions in surface sediment of Wudalianchi. Environ- 
mental Science \& Technology, 2011, 34(9) : 95-99. [ 熊善高, 席北斗, 魏丹等. 五大连池表层沉积物中不同形态磷 的分布. 环境科学与技术, 2011, 34(9): 95-99.]

[23] Sun SQ, Yang J, Tan WC et al. Characteristics of phosphorus release of sediment in East Dongting Lake. Journal of Changsha University of Science and Technology: Natural Science, 2010, 7(2):87-92. [孙士权, 杨静, 谭万春等. 东洞庭湖 底泥的释磷特性. 长沙理工大学学报: 自然科学版, 2010, 7(2): 87-92.]

[24] Han C, Geng JJ, Zhang JA et al. Phosphine migration at the water-air interface in Lake Taihu, China. Chemosphere, $2011, \mathbf{8 2}(6)$ : 935-939.

[25] Geng JJ, Niu XJ, Wang XR et al. The presence of trace phosphine in Lake Taihu water. International Journal of Environmental Analytical Chemistry, 2010, 90(9): 737-746.

[26] Han C, Geng J, Hong Y et al. Free atmospheric phosphine concentrations and fluxes in different wetland ecosystems, China. Environ Pollut, 2011, 159(2): 630-635.

[27] Feng ZH, Song XX, Yu ZM. Distribution characteristics of matrix-bound phosphine along the coast of China and possible environmental controls. Chemosphere, 2008, 73(4) : 519-525.

[28] Sun L. Study on mechanism and process of the phosphine released [Dissertation]. Guangzhou: Guangzhou University, 2012. [孙亮. 磷化氢的释放机理及其过程研究 [学位论文]. 广州: 广州大学, 2012.]

[29] Devai I, Delaune RD. Evidence for phosphine production and emission from Louisiana and Florida marsh soils. Org Geochem, 1995, 23(3) : 277-279.

[30] Feng ZH. Study on the distribution characteristics, release process and transformation mechanism of MBP in marine sediments [Dissertation]. Qingdao: Institute of Oceanology, Chinese Academy of Sciences, 2008. [冯志华. 海洋沉积物中 磷化氢的分布、释放与转化研究 [学位论文]. 青岛: 中国科学院海洋研究所, 2008.]

[31] Yan YP. Adsorption, desorption and precipitation of several soil organic phosphates on iron and aluminum (oxyhydr) oxides [Dissertation]. Wuhan: Huazhong Agricultural University, 2015. [严玉鹏. 几种土壤有机磷在铁铝氧化物表面的 吸附、解吸与沉淀 [学位论文]. 武汉: 华中农业大学, 2015.]

[32] Liu XZ, Li HL, Chen JF et al. The seasonal variation and influence factors of particulate phosphorus across the frontal surface in Changjiang River Estuary. Journal of Marine Sciences, 2011, 29(3) : 88-98. [刘希真, 李宏亮, 陈建芳等. 长江 口跨越锋面颗粒磷季节分布变化特征及影响因素. 海洋学研究, 2011, 29(3) : 88-98.]

[33] Zhang YM, Wang Y, Yang F et al. The spatial distribution and degradation characteristic of phosphorus in suspended particulate matter among different ecological types in Taihu. China Environmental Science, 2016, 36(7): 2128-2138. [张毅 敏, 王宇, 杨飞等. 太湖不同生态型湖区悬浮颗粒磷空间分布和降解速率. 中国环境科学, 2016, 36 ( 7): 2128-2138.]

[34] Reichardt W. Catalytic mobilization of phosphate in lake water and by Cyanophyta. Hydrobiologia, 1971, 38 ( 3/4): 377-394.

[35] Jin XC, Jiang X, Wang Q et al. Seasonal change of $\mathrm{P}$ adsorption/desorption characteristics at the water-sediment interface in Meiliang Bay, Taihu Lake, China. Acta Scientiae Circumstantiae, 2008, 28(1):24-30. [ 金相灿, 姜霞, 王琦等. 太 湖梅梁湾沉积物中磷吸附/解吸平衡特征的季节性变化. 环境科学学报, 2008, 28(1): 24-30.]

[36] He YQ, Shen QR, Kong HM et al. Effect of soil moisture on phosphorus contents of red soil under rice cultivation in aerobic soil condition. Journal of Soil and Water Conservation, 2003, 17(2): 5-8. [何园球, 沈其荣, 孔宏敏等. 水稻旱作 条件下土壤水分对红壤磷素的影响. 土保持学报, 2003, 17(2): 5-8.]

[37] Tang JZ, Haibara K, Toda H et al. Dynamics of ferrous iron, redox potential and pH of forested wetland soils. Acta Ecologica Sinica, 2005, 25(1): 103-107. [ 唐罗忠, 生原喜久雄, 户田浩人等. 湿地林土壤的 $\mathrm{Fe}^{2+}$, Eh 及 $\mathrm{pH}$ 值的变化. 生态学报, 2005, 25(1): 103-107.]

[38] Zhang SJ, Qi QJ, Wang SR et al. Effects of organic matter, manganese and iron on phosphorus fractions and release in the sediments of Erhai Lake. Research of Environmental Sciences, 2011, 24(4) : 371-377. [ 张仕军, 齐庆杰, 王圣瑞等. 洱 海沉积物有机质,铁,锰对磷的赋存特征和释放影响. 环境科学研究, 2011, 24(4): 371-377.]

[39] Li LL. Charateristics of phosphorus distribution and occurrence in soils and sediments of the water-level fluctuation zone in the Three Gorges Reservoir areas [Dissertation]. Chongqing: Southwest University, 2014. [李璐璐. 三峡库区消落带土 壤及沉积物中磷素分布与赋存特征研究 [学位论文]. 重庆: 西南大学, 2014.]

[40] Peng J, Wang B, Song Y et al. Adsorption and release of phosphorus in the surface sediment of a wastewater stabilization 
pond. Ecol Eng, 2007, 31: 92-97.

[41] Luo SS, Wan GJ, Huang RG. Characteristics of distribution and removal of Fe and Mn at the sediment-water interface of Erhai Lake, Yunnan Province. Chongqing Environmental Science, 2000, 22(6) : 19-21. [罗莎莎, 万国江, 黄荣贵. 云 南洱海沉积物一水界面铁、锰的分布和迁移特征. 重庆环境科学, 2000, 22(6): 19-21.]

[42] Liang W, Wang Z, Jiao ZX et al. Study progress on endogenous phosphorus release and its affecting factors. Sichuan Environment, 2012, 31(5) : 105-109. [梁文, 王泽, 焦增祥等. 内源磷的释放作用及影响因素研究进展. 四川环境, $2012,31(5):$ 105-109.]

[43] Zheng LB, Ye Y, Zhou HY et al. Distribution of different forms of phosphorus in seabed sediments from East China Sea and its environmental significance. Oceanologia et Limnologia Sinica, 2003, 34(3): 274-282. [郑丽波, 叶瑛, 周怀阳 等. 东海特定海区表层沉积物中磷的形态、分布及其环境意义. 海洋与湖沼, 2003, 34(3) : 274-282.]

[44] Ahlgren J, Reitzel K, Danielsson R et al. Biogenic phosphorous in oligotrophic mountain lake sediments: differences in composition measured with NMR spectroscopy. Water Research, 2006, 40: 3705-3712.

[45] Ma SL, Ni ZK, Wang SR et al. Organic phosphorus forms in sediments and their relationship with the change of water level in Poyang Lake. Acta Scientiae Circumstantiae, 2016, 36(10): 3607-3614. [马双丽, 倪兆奎, 王圣瑞等. 鄱阳湖沉积 物有机磷形态及对水位变化响应. 环境科学学报, 2016, 36(10): 3607-3614.] 